\title{
An experimental, theoretical and event-driven computational study of narrow vibrofluidised granular materials
}

\author{
Anthony Thornton ${ }^{1, \star}$, Kit Windows-Yule ${ }^{1,2}$, David Parker ${ }^{2}$, and Stefan Luding ${ }^{1}$ \\ ${ }^{1}$ Multiscale Mechanics, Department of Fluid and Thermal Engineering, University of Twente, The Netherlands \\ ${ }^{2}$ School of physics and astronomy, University of Birmingham, UK
}

\begin{abstract}
.
We review simulations, experiments and a theoretical treatment of vertically vibrated granular media. The systems considered are confined in narrow quasi-two-dimensional and quasi-one-dimensional (column) geometries, where the vertical extension of the container is much larger than one or both horizontal lengths. The additional geometric constraint present in the column setup frustrates the convection state that is normally observed in wider geometries.

We start by showing that the Event Driven (ED) simulation method is able to accurately reproduce the previously experimentally determined phase-diagram for vibrofludised granular materials. We then review two papers that used ED simulations to study narrow quasi-one-dimensional systems revealing a new phenomenon: collective oscillations of the grains with a characteristic frequency that is much lower than the frequency of energy injection. Theoretical work was then undertaken that is able to accurately predict the frequency of such an oscillation and Positron Emission Particle Tracking (PEPT) experiments were undertaken to provide the first experimental evidence of this new phenomenon.

Finally, we briefly discuss ongoing work to create an open-source version of this ED via its integration in the existing open-source package MercuryDPM (http://MercuryDPM.org); which has many advanced features that are not found in other codes.
\end{abstract}

\section{Introduction}

Granular materials, large collections of discrete, macroscopic particles, are very common in industry, the natural environment and our everyday lives [1]. Here we focus on vibrofluidised granular materials, where the material is 'fluidised' by a rapidly moving, normally high frequency low amplitude, base-plate that inputs energy into the system. The vibrofluidised system presents a wide range of behaviours, many of which are analogs of behaviours in molecular systems: phase separation [2, 3], Faraday-like pattern formation instabilities $[4,5]$, heap formation and convection [6, 7], segregation [8,9], clustering [10] and periodic cluster expansions [11], etc.

The geometry of the system is known to have a large influence on the dynamics that can be observed within this vibrofluidised system. Here we investigate the effect of changing the system's lateral size and the transition from quasi-two-dimensional to quasi-one-dimensional systems, see Figure 1.

The rest of the paper is laid out as follows: in section 2 we start by introducing the Event Driven numerical methods and, in section 3, show it can be used to accurate reproduce all the known states of a vibrofluidised system. In section 4 we consider the quasi-one-dimensional system,

\footnotetext{
^e-mail: a.r.thornton@utwente.nl
}

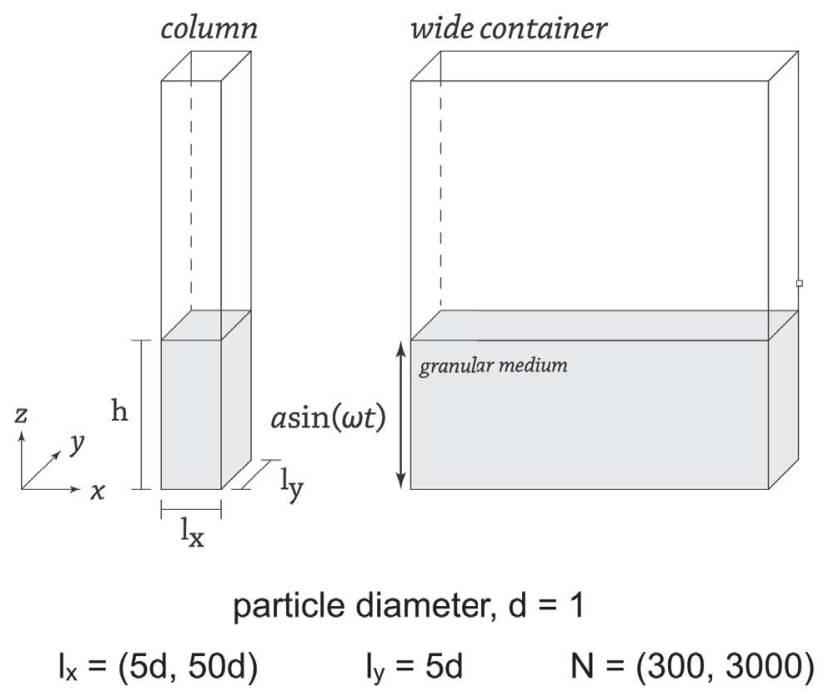

Figure 1. Setup of the geometries that are considered

introducing the new state: the low frequency oscillation (LFO) and (section 5) present both a theory that actually predicts the frequency and positron emission experiments that showed the first experimental evidence of such a state. Finally, in section 6 we draw conclusions and discuss on- 


\section{Time evolution}

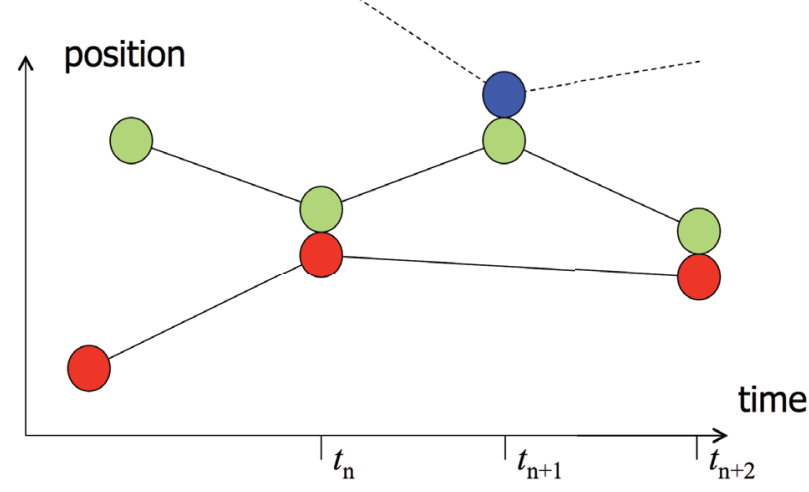

Figure 2. Schematic of how time is advanced in an event driven simulation. From one event $n$ to the next, $n+1$, and so on...

going work to integrate the ED method with the flexible open-source particle solver MercuryDPM.

\section{Event driven simulation of vibrofluidised systems}

In this paper, particle simulations are undertaken using the event driven method $[12,13]$. In this approach, particles move freely under the effect of gravity until an event takes place, i.e. a collision with another particle or a wall. The motion of the particles in between successive events does not have to be simulated, the time is advanced in variable steps, per particle, from one event to the other, see Figure 2. This makes event-driven simulations faster than usual time-stepping simulations, where time is advanced in constant steps. However, the need for an analytical expression for the particle motion is a strong condition that limits the possible interactions between particles. In the following, we consider the most common approach: perfect hard-spheres, which imply binary collisions and no overlap or long-range forces between particles. In brief the ED algorithm works as follows:

1. For each particle compute its trajectory via

$$
\boldsymbol{x}_{i}(t)=\boldsymbol{x}\left(t_{0}\right)+\boldsymbol{v}_{i}\left(t-t_{0}\right)+1 / 2 \boldsymbol{g}\left(t-t_{0}\right)^{2}
$$

and find smallest $t=t_{c}$ for which $\exists i, j: \mid \vec{x}_{i}(t)-$ $\vec{x}_{j}(t) \mid=r_{i}+r_{j}$.

2. Advance particle $i$ and $j$ to $t_{c}$

3. Apply collision rule to update $\boldsymbol{v}_{i}$ and $\boldsymbol{v}_{j}$.

4. Go back to step 1 .

For the considered vibrofluidised system, the whole box (both the bottom and the side walls) is vertically vibrated in a bi-parabolic, quasi-sinusoidal way with a given frequency, $f$ and amplitude $a$. The use of a quadratic instead of a sine function gives a considerable speed advantage in simulations, as the prediction of collision times with the moving walls becomes analytical and thus substantially faster. Full details of the simulations properties used for this paper can be found in [14].

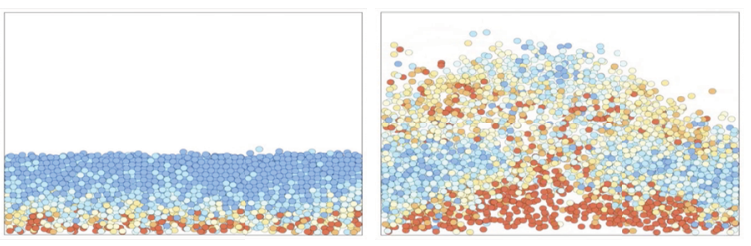

Figure 3. Snapshots of (left) Leidenfrost and (right) convection states from ED simulations. The colour indicates the fluctuating kinetic energy (or granular temperature) of the particles, with red high and blue low.

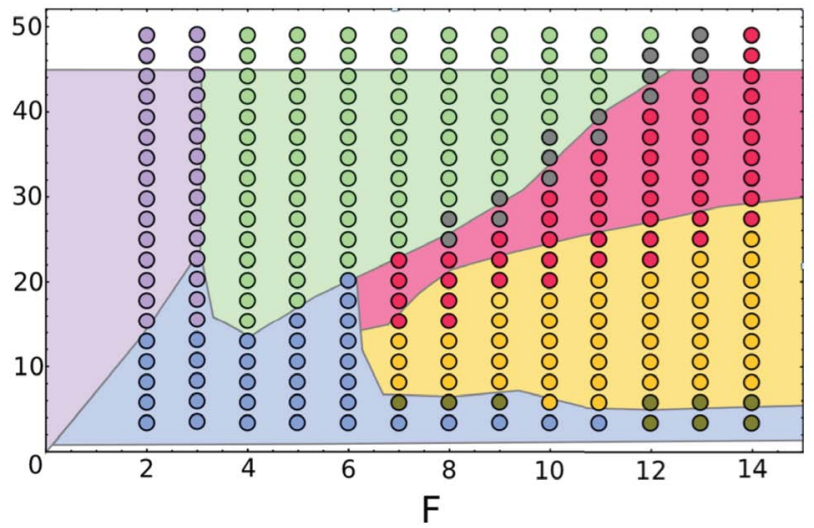

Figure 4. Determination of the states in the vibrofluidised system. States are in blue bouncing bed, yellow undulations. red Leidenfrost, green convection. Dots are from the ED simulations and the background is coloured with data from experiments. The grey circles are transition states.

\section{Phase space from ED}

Vibrofluidised systems are known to exhibit many different states, these include: bouncing bed, bursts, undulations, Leidenfrost, convection and gases [17]. Two of these states as produced in our ED simulations are shown in Figure 3. A phase-space can be constructed demarking the location of these states in terms of the number of filling layers, i.e. the number of particles per unit cross section (parallel to the vibrated base) and either the dimensionless acceleration $\Gamma=A \omega^{2} / g$ or the dimensionless shaking strength $S=a^{2} \omega^{2} / g d$, where $a$ is the amplitude of vibrating base, $\omega$ the angular frequency, $g$ the acceleration due to gravity and $d$ is the dimeter of the particles.

Figure 3 shows a snapshot of a simulation in the Leidenfrost state. This state was first observed in simulations by [15] and takes its name from analogy with the fluid phenomena of the same name in which liquid is suspended over vapour [16]. As indicated by the colours in Figure 3 the granular equivalent is the same with high temperature particles supporting lower temperature particles. As the frequency of the vibrating wall is increased this state transits in to a convection state. The right panel of Figure 3 shows a snapshot of this state where hot particles are rising in the centre and cooling/sinking near the walls.

Figure 4 shows the full phase-space of states as determined by the current ED code compared with the experi- 


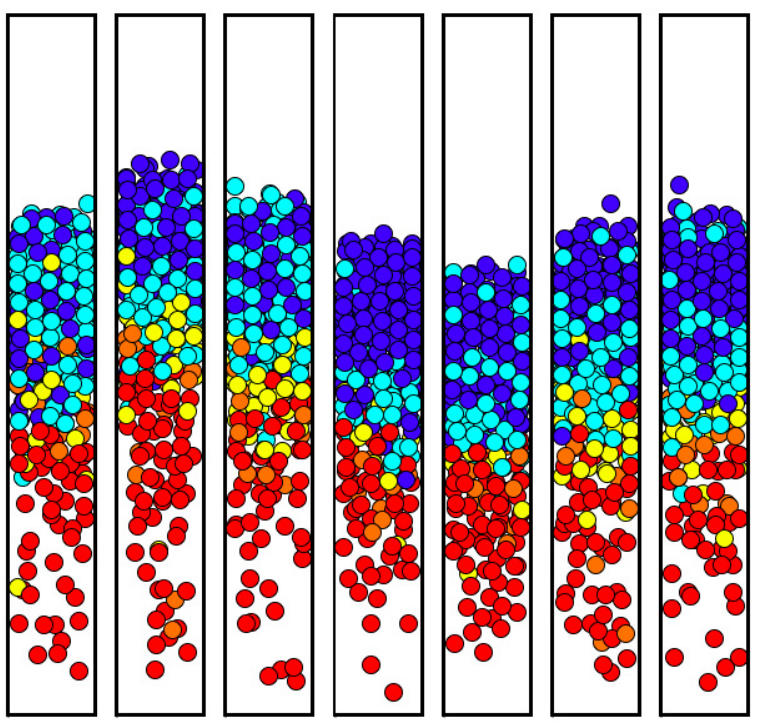

Figure 5. Snapshots of ED simulations of the narrow column. Time progresses from left to right. The time evolution clearly shows an oscillation of the upper surface.

mental and soft particle simulations of [17]. There is excellent quantitative agreement and this validates the use of the event driven method for studying vibrofluidised granular materials. It should be noted that there are a few differences; however, the error is small and could also be in the original data as some of these transitions are hard to exactly determine and experiments can have side effects that can be avoided in simulations. The ED method is several orders of magnitude faster than the soft particles method, in this regime, and gives a lot more information, e.g. granular temperature, than experiments. Therefore this adds a valuable new tool for investigating vibrofluidised granular materials. To emphasis how fast this ED tool is, it should be noted that all the ED data points for Figure 4 were generated on a normal desktop computer within a few hours.

\section{Low frequency oscillations (LFO)}

Using the new ED tool we now turn our attention to narrow column geometry as illustrated by the left schematic in Figure 1. As shown in [14] below a container size of around 10 particle diameters the convection state, illustrated by the right panel of Figure 3, is no longer possible. Therefore in this narrow column geometry it is possible to generate Leindenfrost states at very high accelerations (high $\Gamma$ values) that in wider geometries would have broken into convection, as illustrated in Figure 3.

We next discuss the results of Event Driven (ED) simulations of this frustrated system, that where first reported in [14]. The confined geometry revealed a previous unreported collective oscillations of the grains with a characteristic frequency that is much lower than the frequency of energy injection. A time-series from these frustrated systems is shown in Figure 5 and clearly shows that the top of the gaseous region is not constant but is oscillating in time.

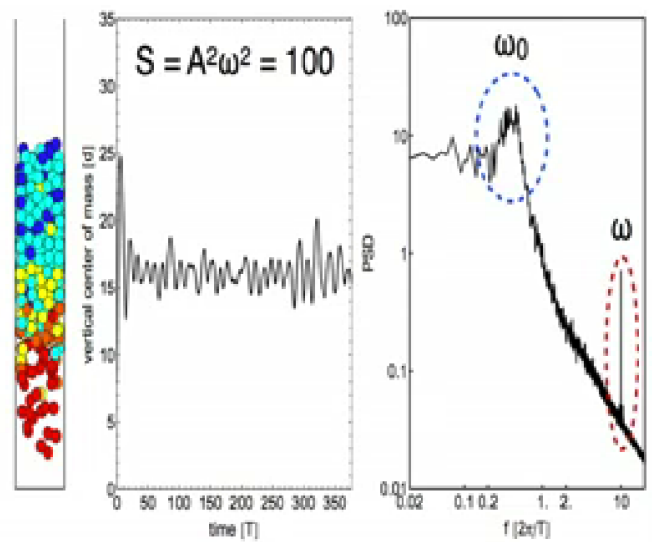

Figure 6. Left: Snapshots of ED simulations of the narrow column. Middle: Centre of mass of the column against time. Right: Fourier transform of the centre of mass. $w_{0}$ is the characteristic low frequency oscillation and $\omega$ is the driving frequency on the vibrating plate.

Careful analysis of the centre-of-mass of the narrow column system revealed a characteristic frequency in these oscillations that is much lower than the driving frequency: the LFO. Figure 6 shows a typical time-trace of the centreof-mass of the corresponding Fourier transform, you can clearly see a peak at a considerably lower frequency than the driving frequency of the plate: the low-frequency oscillations (LFOs). In [14] the frequency and amplitude of these oscillations was studied as a function of the energy input parameters and the size of the container. It was later observed that, in the quasi-two-dimensional setup, LFOs are present even in the convective regime. This suggests that they may play a significant role in the transition from a density inverted state to convection. This transition and the role LFOs play in it was further studied by Rivas $e t$. al. [18] and the fluctuations due to the finite number of particles.

\section{Comparison with theory and experiments}

In [14] two theoretical models are also presented; the first one, based on Cauchy's equations, is able to predict with high accuracy the frequency of the particles collective motion. The model shows that a sufficient condition for the existence of the low-frequency mode is an inverted density profile with distinct low and high density regions, a condition that may apply to other systems too. The second, simpler model just assumes an harmonic oscillator like behaviour and, using thermodynamic arguments, is also able to reproduce the observed frequencies with high accuracy. For the details of both models please see [14].

In [20] Positron Emission Particle Tracking (PEPT) was used to experimentally observe and study the novel LFOs. PEPT is a noninvasive technique whereby a single particle, physically identical to the others in the system, is labeled with a beta-plus-emitting isotope. The back-toback pairs of gamma-rays emitted due to the rapid annihi- 

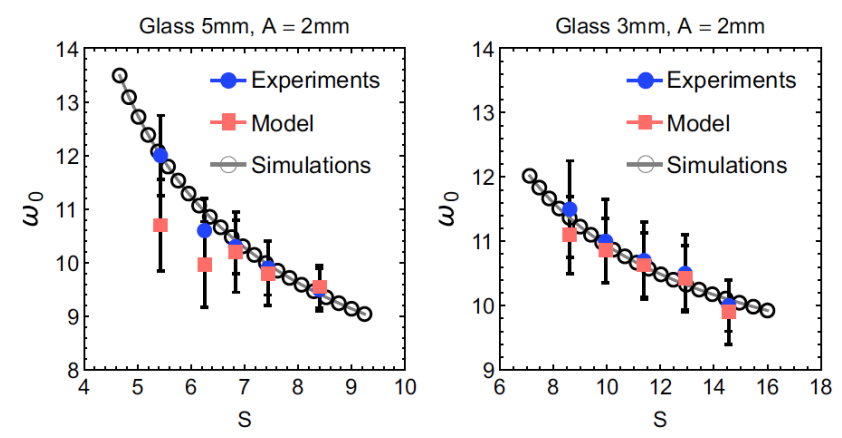

Figure 7. Experimental and theoretical values of $\omega_{o}$ as a function of the shaking strength $S=a^{2} \omega^{2} / g d$. Data for $d=3 \mathrm{~mm}$ and $d=5 \mathrm{~mm}$ glass particles, for $A=2 \mathrm{~mm}$. Originally published in [19] and reproduced wth permission.

lation of positrons with electrons within this tracer particle are detected using a dual-headed gamma-camera, and can be used to triangulate its position with millimetre precision and millisecond time resolution. Figure 7 shows the comparison between the model, experiments and the simulations across a range of shaking strengths $S$ for two differently sized glass particles. Where possible the known particle values were used in the simulations and the theoretical work. The experimentally acquired results show a close qualitative and quantitative agreement with both theory and simulations across the range of parameters tested. The agreement between all three (experiments, simulations, and theory) is always within $10 \%$ and improves as the value of the shaking strength $S$ is increased. This makes sense as the model is only formally valid in the high shaking strength, $S$, limit and in this limit the agreement is very good. This was also tested for other materials and parameters and the agreement was always found to be good, illustrating that the LFOs are a robust, reproducible phenomena.

\section{Conclusions}

We have shown that event driven simulations are a valid tool for investigating vibrofluidised systems. The ED tool is so quick it is able to simulate thousands of particles in real time meaning you are able to interactively try new ideas. This is how the phenomenon of the low frequency oscillations was discovered in the first place. This highlights how new developments in computational tools can lead to new physical understanding that would have been hard without the rapid interactive feedback. The tool described in this paper has been used so far in various publications; for example[14, 18-20]. Work is underway to integrate this rapid ED tool with the open-source particle solver MercuryDPM (http://MercuryDPM.org) [21, 22]; which has many advanced features that are not found in other codes that could be integrated with this rapid eventdriven code.

\section{Acknowledgements}

This work was financially supported by the NWO-STW Vidi Grant No. 13472 and NWO-STW Vici Grant No. 10828.

\section{References}

[1] H.M. Jaeger, S.R. Nagel, R.P. Behringer, Physics Today 49 (1996)

[2] J.S. Olafsen, J.S. Urbach, Phys. Rev. Lett. 81, 4369 (1998)

[3] P. Melby, F.V. Reyes, A. Prevost, R. Robertson, P. Kumar, D.A. Egolf, J.S. Urbach, Journal of Physics: Condensed Matter 17, S2689 (2005)

[4] F. Melo, P. Umbanhowar, H.L. Swinney, Phys. Rev. Lett. 72, 172 (1994)

[5] S. Luding, E. Clement, J. Rajchenbach, J. Duran, EPL (Europhysics Letters) 36, 247 (1996)

[6] S.G.K. Tennakoon, R.P. Behringer, Phys. Rev. Lett. 81, 794 (1998)

[7] M. Medved, Phys. Rev. E 65, 021305 (2002)

[8] K. Ahmad, I.J. Smalley, Powder Technol. p. 69-75 (1973)

[9] A. Kudrolli, Reports on Progress in Physics 67, 209 (2004)

[10] S. Luding, H.J. Herrmann, Chaos 9 (1999)

[11] N. Rivas, S. Ponce, B. Gallet, D. Risso, R. Soto, P. Cordero, N. Mujica, Phys. Rev. Lett. 106, 088001 (2011)

[12] B.D. Lubachevsky, Journal of Comp. Phys. 94 (1991)

[13] S. Luding, S. McNamara, Granular Matter 1 (1998)

[14] N. Rivas, S. Luding, A.R. Thornton, New J. Phys. 15, 113043 (2014)

[15] B. Meerson, T. Pöschel, Y. Bromberg, Phys. Rev. Lett. 91 (2003)

[16] J.G. Leidenfrost, International Journal of Heat and Mass Transfer 9, 1153 (1966)

[17] P. Eshuis, K. Weele, van der, D. Meer, van der, R. Bos, D. Lohse, Phys. Fluids 19 (2007)

[18] N. Rivas, A.R. Thornton, S. Luding, D. van der Meer, Phys. Rev. E 91, 042202 (2015)

[19] C.R.K. Windows-Yule, N. Rivas, D.J. Parker, A.R. Thornton, Phys. Rev E 90(6) (2014)

[20] C.R.K. Windows-Yule, N. Rivas, D.J. Parker, Phys. Rev. Lett. 111, 038001 (2013)

[21] A.R. Thornton, D. Krijgsman, A. te Voortwis, V. Ogarko, S. Luding, R. Fransen, S. Gonzalez, O. Bokhove, O. Imole, T. Weinhart, A review of recent work on the Discrete Particle Method at the University of Twente : An introduction to the opensource package MercuryDPM, in DEM 6: Conf. Proc. (2013), pp. 393-399

[22] A.R. Thornton, D. Krijgsman, R. Fransen, S. Gonzalez, D. Tunuguntla, A. te Voortwis, S. Luding, O. Bokhove, T. Weinhart, Newsletter EnginSoft 10, 48 (2013) 